\title{
Communicating between Healthcare Disciplines: An Educator's Perspective
}

\author{
Lauren Boardman* \\ College of Coastal Georgia, USA
}

Submission: May 29, 2017; Published: June 27, 2017

*Corresponding author: Lauren Boardman, College of Coastal Georgia, USA, Email: lboardman@ccga.edu

Abstract

Introduction: In an ever-changing, fast-paced, healthcare industry practitioners are expected to keep up with the demands placed upon them. Students step out of a sheltered silo of healthcare disciplines not often realizing the importance of interprofessional communication or role appreciation of their fellow team members.

Purpose: This paper serves as an educator's perspective of creating a simulation for students from the disciplines of nursing, radiological, and surgical technology. Participating in a shared learning experience with multiple healthcare disciplines is an important means of breaking professional silos; affording students to practice interprofessional communication and develop role appreciation outside their practice of study.

Conclusion: Teamwork and collaboration are important factors educator's must incorporate into curriculum to prepare students for the realities faced in clinical practice. As educators and practitioners, we serve as gatekeepers to the clinical world. How we communicate and collaborate should reflect the values and principles expected of the student. Therefore, planning and implementing shared learning experiences between healthcare disciplines can be an enriching for both students and educators.

Keywords: Interprofessional; Simulation; Collaboration; Teamwork; Shared learning; Allied health; Nursing; Educators; Communication

Abbreviations: IEP: Interprofessional Education; RIPLS: Readiness for Interprofessional Learning Scale; IEPS: Interprofessional Education and Perception Scale

\section{Introduction}

All too often healthcare students fail to realize the important role they will play as a member of the healthcare team. During education their focus is to learn as much about their profession and practice before graduation and licensure examination. Exposure to other disciplines may be limited to clinical experience and observation while rotating through various units and specialty areas of practice. As a result, educators often focus on preparing students for practice by way of training enabling them to be successful in licensure; forgoing the application of didactic and laboratory scenarios encountered in professional practice [1]. A novice practitioner encountering such events may retreat even further into their own professional culture, interacting only when absolutely necessary. Incongruent care and negative health outcomes may result from this behavior and are attributed to the breakdown of communication and collaboration between members of the healthcare team.

\section{Review of current evidence supporting interprofessional education (IEP)}

The Institute of Medicine (IOM) published the groundbreaking report To Err Is Human over a decade ago making a decisive statement that the quality and safety of the American healthcare system was in shambles, endangering and contributing to sentinel events of nearly 98,000 clients yearly [2]. The position of the report stated healthcare providers lack the skills to effectively collaborate between disciplines, leading to communication breakdown, professional error, and poor client outcomes. The IOM attributed these adverse events as a result of training healthcare professionals in isolation, contributing to a community of practitioners with limited knowledge of the importance of collaborative practice and understanding of other healthcare provider's professional role(s). The termed to describe this archaic system of education is a "professional silo."Professional silos often foster relationships 
based on power, competition, and hierarchies, resulting in inadequate preparation for teamwork [3]. When cultures clash, opportunities for miscommunication and errors arise [4]. The danger in continuing this educational cycle is the potential for stereotyping which further contributes to a disconnect between healthcare professionals, undermining the unique knowledge and skill set each discipline brings to practice.

If professional silos and stereotyping are knowingly created by healthcare disciplines, how then do we address the breakdown of these barriers? Students learn from their mentors, instructors, faculty, preceptors, and by observing others in practice. If key educational players exhibit behavior maintaining isolative practice, then change cannot take place. Hierarchies are another culprit making communication and collaboration a difficult task between practitioners [5]. The question educators should ask is, "how do we tackle this complicated issue?" One solution is to implement shared learning experiences into healthcare curriculums. Interprofessional education (IPE) is just one method of shared learning which can be used to bring healthcare disciplines together. IPE has been defined as members or students of two or more professions engaged in learning with, from, and about each other [6]. There has been no consensus as to when IPE should first be implemented, but it has been suggested students in an undergraduate healthcare program may glean the most benefit from implementing this type of learning as it fosters positive attitudes to collaboration early in a student's professional life and can assist in pre-empting negative stereotypes of other professions [7].

Barriers continue to exist preventing the implementation of IPE both in clinical practice and in academia [1]. Issues arise with scheduling of courses and lab times when students from multiple healthcare disciplines may come together and engage in a shared learning environment. Educators are responsible to collaborate with each other and make the effort to plan shared learning activities for students. Simply discussing the importance of communication during lab, in clinical, or didactic does not leave the same impact as actually experiencing communication between healthcare providers. Just as we are encouraged to cultivate a culture valuing evidence-based practice, so should healthcare educators seek to create a culture valuing IPE. A lingering set-back to implementing IPE, like evidence-based practice, seems to be lack of knowledge and how to implement shared learning for students.

Learning about IPE for educators is one challenge and actualizing IPE is another. There are a number of colleges and universities where students exist in isolation never crossing paths during their respected programs of study. Changing existing practice of isolative learning requires partnerships of teaching institutions and delivery systems to create shared learning environments and requiring educators to model interprofessional collaborative practice [8]. Implementing IPE can be an enriching experience for both educators and students; breaking down the walls set between professionals and aiding in collaboration, communication, and role understanding and appreciation. This report seeks to align the concepts presented in the literature review with the implementation of an interprofessional simulation and discussion between faculty members, and students, of each discipline participating in the project.

\section{Methods}

\section{Design}

A mixed method, pre-test/post-test, design was selected using paper and pencil instruments, a brief demographic survey, and an interprofessional simulated experience. The Readiness for Interprofessional Learning Scale (RIPLS) and the Interprofessional Education and Perception Scale (IEPS) served as the two main evaluation tools, while a brief demographic survey was used to determine identify cohort characteristics that may have influenced pre and pot-testing scores. Nursing students provided feedback to the researcher through a structured debriefing following implementation of the interprofessional simulation. Data from radiological and surgical technology students were not directly collected. An educator's perspective was the method in which feedback from allied health disciplines were collected. These were designed as semistructured interviews with the directors from both disciplines; a brief semi-structured interviewfollowing simulation and a formal, structured, interview one month following project implementation.

\section{Participants}

Participants were students from the allied health fields of surgical and radiological technology and nursing students enrolled full-time in an undergraduate associate degree program in the United States. Convenience sampling was used as the method for participant selection in nursing. Radiological and surgical technology students were asked to participate, at will, by the directors of their programs. Students from these disciplines were granted clinical hours (radiology technology) or were purely on a volunteer basis (surgical technology). Nursing was the only discipline included in sampling related to measurement and analysis for this project.

\section{Ethics}

Institutional Review Board approval was granted prior to the start of the project by the university where the researcher was completing their doctor of nursing practice degree and the institution in which the simulation was taking place.

\section{Procedure}

The interprofessional simulation was devised through the combined efforts of the researcher and directors from both the surgical and radiological technology programs. Students participated in a simulation addressing the care of an elderly client with a right hip fracture. Nursing students began their rotation in the simulation lab completing the pre-operative 
checklist, a nursing assessment, client education, verification of signed consent, medication reconciliation, and administration of pre-operative medications using a high-fidelity simulator. Students then transported a static mannequin by cart to the radiology department where they met the radiology students for pre-operative $\mathrm{x}$ rays. Radiological and nursing students collaborated while giving report, during client transfer on and off the exam table, and after the x-ray picture was approved so the client could proceed to surgery.

Next, nursing students transported the same mannequin to the operating room suite where they met other nursing students playing the role of the circulating and operating room nurses. Transport nurses provided a hand-off report detailing the client's diagnosis and past history, completion of x-rays, and therapies administered prior to traveling, to the operating room staff. Operating room nursing students were expected to communicate and collaborate with surgical technology students, the surgeon and anesthesiologist. Nursing students in the operating room were surprised with two potential complications: a client allergy to the prescribed intravenous antibiotic, and a retained sponge at the end of surgery.

Following simulation nursing students completed post-test tools and a focused debriefing lead by the researcher and lead instructor of the course. Student feedback was recorded by the researcher as anecdotal data to supplement quantitative results from pre-test and post-test instruments. One-month follow-up post-testing was completed by nursing students. At this time the researcher scheduled interview sessions with both the directors from allied health programs, with the goal of obtaining feedback from their students' debriefings following simulation.

\section{Instruments}

Effects of simulation were measured using student scores on the IEPS and the RIPLS. Both tools are public domain. The IEPS by Leucht, Madsen, Taugher, and Petterson is an 18-item instrument using a 6-point Likert scale ranging from 1 (strongly agree) to 6 (strongly disagree) [9]. The scale measures the four attitudes of professional competency and autonomy; perceived needs for professional cooperation, perception of actual cooperation, resource sharing within and across professions, and understanding the value and contributions of other professionals and professions [10]. The total reported Cronbach's alpha for reliability for the IEPS is 0.87 . This tool was administered twice; once with the pre-test, then with post-testing.

The RIPLS, created by Parsell and Bligh, assesses the readiness of students for interprofessional learning [10]. It is a three-factor ,19 item tool, using a 5-point Likert scale with reverse scoring from 1 (strongly agree) to 5 (strongly disagree). Internal consistency is established by a Cronbach's alpha value of 0.90 for the entire 19 item scale.Construct validity for the IEPS and RIPLS is demonstrated through the use of repeated factor analysis to refine questions, and each scale's content is rooted using interprofessional theory as a basis for questionnaire development (MacKay, 2004). This tool was administered twice; once with the pre-test, then with post-testing.

The demographic survey was a descriptive tool created by the researcher, and is a five question tool seeking to collect the following data: age, gender, highest educational degree, prior employment in the healthcare industry, and position of employment if working in the healthcare industry. This survey was completed once with pre-testing tools.

\section{Results}

\section{Nursing}

Pre-test IEPS and RIPLS indicated students had an overall positive outlook on IPE and understanding of the importance for implementing these types of exercises in an undergraduate nursing curriculum. There was a unanimous agreement nurses need to cooperate with other healthcare disciplines making every effort to understand the capabilities and contributions of other professionals. Students believed shared learning with allied health students would make them a more effective team member, improve communication and relationships between healthcare professionals, and clients would benefit from shared learning experience involving problem solving between healthcare students. Immediate post-test findings indicated a decrease in scores with questions relating to how other professionals viewed nurse's contributions, if nurses are in fact respected by other professionals, and nurse's work being dependent upon the work of other professionals. One month later however, scores improved between post-test one and two. The majority of students again agreed nursing is respected by other healthcare professionals and nurses do make positive contributions to the interprofessional team (Table 1). The one score that did not improve as hoped was the belief that nurses work depends on the work of other healthcare professionals. This may be to the hierarchical nature the way healthcare disciplines are educated, with the physician at the top of the pyramid, followed by the nurse practitioner, physician assistant, registered nurse, and so on [11].

The nursing debriefing completed following simulation was valuable in gaining the student lived perspective with IPE. Student nurses were also requested, via email, to provide the researcher with anecdotal experiences during simulation. Overall responses from nursing students were positive (Table 2). During debriefing, students reported they would like to have this experience again during their education; that engaging in IPE experiences helps to build confidence in communication skills with individuals in other healthcare professions. Students enjoyed engaging with allied health students, reporting how they had never thought they could have had such a great learning experience with students from another department. Debriefing continued with a positive tone, and the common consensus was to include this experience in nursing curriculum; possibly 
incorporating interprofessional simulation in the first medical surgical course and again in their senior term. This finding coincides with the literature review concerning the appropriate timing in curriculum to introduce IPE [7].

The demographic survey was included to gain insight into if student age, profession, and education may have contributed to scoring on tools and performance during the simulation exercise (Table 1). The mean age was 35.3, with the majority of students being women $(85 \%)$. Seventy percent of nursing students had previously completed a degree from a trade/vocational program, $15 \%$ recorded some college courses, $5 \%$ had a high school diploma, and $10 \%$ reported having a bachelor's degree. Another area of interest was prior employment in healthcare; $95 \%$ of students responded past experience or current employment in the healthcare field. For those reporting working in the healthcare field, 65\% were employed as licensed practical nurses (LPN's) and $30 \%$ as nursing assistants. This was a unique characteristic of this nursing cohort that was taken into consideration during analysis of findings.

\section{Allied health}

The interviewing process consisted of face-to-face interviews between the researcher and directors for the radiological and surgical technology programs. Semi-formal and formal interviews were conducted separately with the directors from allied health disciplines. A positive overtone from directors was collected; both felt students enjoyed the experience and shared learning experiences should be done as often as possible (Table 2).

The director of the radiological technology program was delighted to report all students showed up to be a part of the simulation. The director continued to discuss how beneficial the experience was; how students were forced to prioritize for client care in transferring the client with a broken hip, best positioning to achieve the clearest image, and how great this type of simulation was for students to experience a trauma situation prior to starting clinical rotation.

The director from surgical technology was pleased to report the experience was helpful in integrating theory to practice for their students, more importantly the simulation afforded their students the opportunity to guide nursing students in the OR environment where they have no previous experience. Leadership and communication are achievable outcomes for the surgical technology student while participating in IPE activities.

Table 1: Demographic Data of Nursing Students.

\begin{tabular}{|c|c|c|c|c|}
\hline Age of Participant & Gender & $\begin{array}{c}\text { Highest Level of School } \\
\text { Attained }\end{array}$ & $\begin{array}{c}\text { Prior Employment/ } \\
\text { Experience in Healthcare }\end{array}$ & $\begin{array}{c}\text { Level of Position Held in } \\
\text { Healthcare Field }\end{array}$ \\
\hline Mean= 35.3 years & $\begin{array}{c}\text { Female }=17(85 \%) \\
\text { Male=3(15\%) }\end{array}$ & $\begin{array}{c}\text { Trade/vocational degree= } \\
14(70 \%) \\
\text { Some College }=3(15 \%) \\
\text { High School Diploma= } \\
1(5 \%) \\
\text { Bachelor's Degree= } \\
2(10 \%)\end{array}$ & $\begin{array}{c}\text { Yes }=19(95 \%) \\
\text { No }=1(5 \%)\end{array}$ & $\begin{array}{c}\text { None }=1(5 \%) \\
\text { Licensed Practical Nurse } \\
(\text { LPN })=13(65 \%) \\
\text { Nursing Aide }=6(30 \%)\end{array}$ \\
\hline
\end{tabular}

Table 2 : Student and Faculty Interview/Debriefing Responses to Interprofessional Simulation.

Faculty Interview Responses

Radiology Technology Director: "All my students showed up; I was amazed! They were so excited to be a part of the project. This is an activity (interprofessional simulation) we should consider making a requirement of all healthcare students educational experience."

"Working together to plan the simulation was a great way for educators to get together and learn a little more about each other."

\section{Nursing Student Debriefing Responses}

Student \#1: "I think that this is an important thing to do (simulation) with other students. We don't always get this experience in clinical."

Student \#2: “For those of us working as LPN's, we do get experience talking with other professionals but I agree that for those that don't this is a valuable experience." 
Researcher's Personal Reflection: "The reaction of both directors made
it even more apparent interprofessional opportunities and a shared
learning environment benefit all healthcare students. Even though the
focus was on nursing it was interesting to hear the other professions
had their own outcomes that were achieved through this experience."

"Planning and implementing this simulation was a wonderful experience that helped all educators involved break outside our defined discipline. It was educational to hear each person discuss what their students' skill sets were and what was required for them to meet simulation objectives."

"The directors and I worked so well together. It just goes to show when the focus is on student success, different disciplines can come together and do something great!"
Student \#3: "Communication is key when working with other healthcare professionals...working together as a team is hard if everyone is on different pages."
Student \#4: "This simulation made us look ahead as to what was going to be needed; like IV access and what was the most pertinent information needed in handoff between departments."

Student \#5: "The OR was intense. At first I thought the students were a little rude, but then after talking I understand they are the expert in that environment; like we as a nurse would be serious about caring for our clients."

\section{Discussion}

\section{Data interpretation}

Quantitative data from paper and pencil tools was verified after analyzing qualitative reports from both nursing, allied health students, and allied health directors. Students from all disciplines identified this exercise was beneficial to build their communication and collaboration skills. Even though results from the initial post-testing, nursing student's opinion of IEP and the roles of the healthcare team rebounded positively. This may be attributed to time for reflection on the initial "aftershock" and adrenaline release of the trauma-based simulation.

Students from radiological technology reported a positive and energetic response to simulation. The director made sure to reiterate this exercise was optional and not a required activity of their courses. Students had never experienced simulation with other healthcare students and were excited to participate. An unanticipated finding from the interview was the experience contributed to students achieving outcomes of their educational curriculum. Students were afforded a simulated "real-life" experience they might encounter in practice. The director reported students enjoyed the experience and would like to see this incorporated into their courses, and reported a desire to understand IPE more thoroughly so in planning for the next simulation they would be able to formulate clear objectives for their students.

Not all surgical technology students participated in the simulation experience and were recruited by the director. Senior students were asked to act as the technician during simulation because they had the most experience and could direct nursing students when needed. The director reported this experience was valuable for their students because they were able to practice communication skills with students outside of their future profession and share their expert knowledge with them. One of many objectives was to break professional silos healthcare students are educated in. In the operating suite both surgical technology and nursing students shared their knowledge to transition the client safely through the surgical procedure. Nursing students experienced a retained sponge after site closure leading them to collaborate with the surgical technician, circulating nurse, and surgeon to determine the best course of action while protecting the client from infection or hemodynamic instability during reopening and closure of the surgical site. Students also had the opportunity to identify an antibiotic allergy and communicate this to the surgical team prior administering the medication to the client.

The overall tone both educators (directors) expressed was one of positivity towards IPE. The potential role IPE plays in the development of interdisciplinary appreciation and collaboration is endless. Part of each interview was a discussion of how educators in different healthcare disciplines should share a sense of comradery. The best way to develop comradery is to respect and have open dialogue with educators in your institution; including educators from other disciplines. Implementing IPE into a healthcare curriculum makes a bold statement that educators see one common goal to align disciplines; this is the welfare of the client.Adopting an IPE learning platform requires a partnership of multiple stakeholders (institutions, care delivery systems) and educators willing to model interprofessional collaborative practice [8].

\section{Limitations}

There are areas identified as limitations. The first imitation identified was the attrition rate of nursing students. Attrition for the medical surgical course selected has been an issue. This course was selected for the IPE project because students 
had little experience with simulation, clinical, or the concept of interprofessional collaboration and communication. There is no identifiable solution to prevent students dropping the course by midterm.

A second limitation was sample size. A larger sample of students would lead to a more robust data analysis and findings. An interesting finding was the number of students in the cohort currently working in healthcare; $65 \%$ of students reported current employment as LPNs and 30\% working as a nurse's aide. The institution the project was implemented in houses both an associate degree nursing and licensed practical nursing program. After completion of their LPN many students enroll into the associate degree program. This is not believed to discredit findings but was an important consideration from the data analysis. Replicating the project at an institution offering a bachelor in nursing degree may eliminate the issue because often students enrolled in such programs are seeking a traditional four-year degree, are younger in age, and less likely to have prior experience in the healthcare industry.

The final limitation was collecting data solely from nursing students. Future replication should include feedback from allied health and nursing students. Interviews with the allied health directors provided an opportunity to gain insight from an educator's perspective as to how the simulation experience aided in educating their students, but data collection from all three disciplines would contribute to a more comprehensive evaluation of how shared learning through interprofessional simulation is perceived by all involved.

\section{Conclusion}

From an educator's perspective, this project was a learning experience which will be valuable for further IPE endeavors. The implementation of the simulation shed more light on the positive impact IPE experiences afford healthcare students, and was a learning opportunity for all faculty members involved. Though the focus was aimed at improving collaboration and communication for nursing students, the project also indirectly achieved the same outcomes for educators in allied health.

In the literature review emphasis is expressed on the educator's responsibilities for demonstrating an attitude of openness towards collaboration and shared learning. This is a critical area to develop for the future of IPE. The experience of designing, planning, and evaluating the effects of this project demonstrates how faculty from different disciplines play a crucial role in socializing students and preparing them to enter practice as a collaborative team member. It is the responsibility of faculty working in a multidisciplinary environment to be role models. How educator's treat one another may indirectly influence attitudes and opinions of our students; in response developing professional stereotypes and taking this into practice as a new graduate. Through the combined expertise of educator's, our students observed three leaders from different disciplines planning a shared learning experience to improve collaboration between healthcare providers. This experience has broken professional silos existing in the institution and laid the foundations and desire to continue collaboration between allied health and nursing.

In planning the simulation we experienced issues presented in literature. Finding times to meet and plan IPE was challenging but necessary for each educator to contribute ideas of what elements could be added into the scenario to capitalize on the unique skill sets of each discipline. Building relationships with the directors from allied health has opened the door for further collaboration. Breaking the silos in a small healthcare institution may seem a trivial conquest, but was an important barrier to implement and develop a shared learning model for all curriculums involved.

In closing, it cannot be emphasized enough how important educator's roles are in creating a culture of IPE in institutions of higher education. Simulation is a valuable mode for IPE and shared learning. The more realistic the scenario the better reported outcomes from students. Educators must make effort to be involved in collaborative processes with members of other healthcare disciplines when opportunity arises. To continue to silo undergraduates is irresponsible and will not improve health outcomes. It is the responsibility of educators to "practice what we preach": we need to be willing to demonstrate appreciation for collaborative relationships with other disciplines and lead by example. No student can afford to have their first interprofessional experience be their first day on a nursing unit.

\section{References}

1. Jukkala A, White M (2014) The continued need for interprofessional collaboration and research. Appl Nurs Res 27(2): 95-96.

2. (2003) The Institute of Medicine. To Err is human. Institute of Medicine.

3. McNair R (2005) The case for educating healthcare students in professionalism as the core content of interprofessional education. Med Educ 39(5): 456-464.

4. Engum S, Jeffries P (2012) Interprofessional collisions: Bringing healthcare professions together. Collegian 19(3): 145-151.

5. Lydon A, Zlatnik M, Wachter R (2011) Effective physician-nurse communication: A patient safety essential for labor and delivery. Am J Obstet Gynecol 205(2): 91-96.

6. Bridges D, Davidson R, Odegaard P, Maki I, Tomkowiak J (2011) Interprofessional collaboration: Three best practice models of interprofessional education. Med Educ Online 16(6035): 1-10.

7. Brown T, Palmero C, McKenna L, Boyle M, Williams B, et al. (2012) Are undergraduate health care student 'ready' for interprofessional learning? A cross-sectional attitudinal study. The Internet Journal of Allied Health Sciences and Practice 10(3): 1-11.

8. (2013) The Josiah Macy Jr. Foundation. Transforming Patient Care: Aligning Interprofessional Education with Clinical Practice Redesign. The Josiah Macy Jr. Foundation, Conference Recommendations, USA, pp. 1-11. 
9. Leucht R, Madsen M, Taugher M, Petterson B (1990) Assessig professional perceptions: Design and validation of an interdisciplinary education perception scale. J Allied Health 19(2): 181-191.

10. MacKay S (2004) The role of perception questionnaire (RPQ): A toll for assessing undergraduate students' perceptions of the role of other professionals. J Interprof Care 18(3): 289-302.
11. Delunas LR, Rouse S (2014) Nursing and medical student attitudes about communication and collaboration before and after an interprofessional education experieince. Nurs Educ Perspect 35(2): 100-105.
This work is licensed under Creative Commons Attribution 4.0 License
Your next submission with Juniper Publishers will reach you the below assets

- Quality Editorial service

- Swift Peer Review

- Reprints availability

- E-prints Service

- Manuscript Podcast for convenient understanding

- Global attainment for your research

- Manuscript accessibility in different formats

( Pdf, E-pub, Full Text, Audio)

- Unceasing customer service

Track the below URL for one-step submission https://juniperpublishers.com/online-submission.php 